\title{
Serum myostatin levels are associated with abdominal aortic calcification in dialysis patients
}

\author{
Su Mi Lee ${ }^{1, *(1)}$, Seong Eun Kim ${ }^{1, *(1)}$, Ji Young Lee ${ }^{2(\mathbb{D}}$, Hyo Jin Jeong ${ }^{3(\mathbb{D}}$, Young Ki Son ${ }^{1(\mathbb{1})}$, Won Suk An ${ }^{1(\mathbb{D})}$ \\ ${ }^{1}$ Department of Internal Medicine, Dong-A University College of Medicine, Busan, Republic of Korea \\ ${ }^{2}$ Department of Internal Medicine, Busan Veterans Hospital, Busan, Republic of Korea \\ ${ }^{3}$ Department of Internal Medicine, Dong-Eui Medical Center, Busan, Republic of Korea
}

\begin{abstract}
Background: Serum myostatin levels are increased according to renal function decline and myostatin may be a main mediator of chronic kidney disease-related sarcopenia. A previous study reported that serum myostatin level was negatively associated with abdominal aortic calcification (AAC) in older males. The aim of this study was to assess the association between serum myostatin level and AAC among dialysis patients of both sexes. In addition, we analyzed the relationship between serum myostatin level, muscle mass, and bone mineral density (BMD).

Methods: In this cross-sectional study, we evaluated AAC in the lateral lumbar spine using plain radiography and BMD in 71 patients undergoing dialysis. We classified patients into two groups according to the median value of myostatin as follows: those with high myostatin levels $(\geq 5.0 \mathrm{ng} / \mathrm{mL})$ and those with low myostatin levels $(<5.0 \mathrm{ng} / \mathrm{mL})$. Results: The proportion of patients with an AAC score of five points or more was higher among those with low myostatin levels. Myostatin level was negatively associated with AAC scores on plain radiography and had a positive association with skeletal muscle mass and T-scores for BMD measured at the total hip and femur neck. Lower myostatin levels were independently associated with higher AAC scores following adjustment for age, sex, diabetes mellitus, dialysis vintage, dialysis modality, and osteoprotegerin level.

Conclusion: Lower serum myostatin levels were associated with higher AAC scores, lower muscle mass, and lower BMD in dialysis patients. Further, prospective studies and those with larger cohorts are necessary to validate these findings.
\end{abstract}

Keywords: Bone density, Dialysis, Muscle, Myostatin, Vascular calcification

Received February 17, 2019; Revised July 1, 2019;

Accepted July 3, 2019

Edited by Young-Ki Lee, Hallym University, Seoul, Republic of Korea

Correspondence: Won Suk An

Department of Internal Medicine, Dong-A University College of Medicine, 32 Daesingongwon-ro, Seo-gu, Busan 49201, Republic of Korea.E-mail:anws@dau.ac.kr

*Su Mi Lee and Seong Eun Kim contributed equally to this work.

Copyright (c) 2019 by The Korean Society of Nephrology

(a) This is an open-access article distributed under the terms of the Creative Commons Attribution Non-Commercial License (http://creativecommons. org/licenses/by-nc-nd/4.0/), which permits unrestricted non-commercial use, distribution, and reproduction in any medium, provided the original work is properly cited.

\section{Introduction}

Chronic kidney disease (CKD) is a public health problem, with a prevalence that is steadily increasing. The high prevalence rate of CKD is explained by the increased proportion of elderly people, hypertension, and diabetes in the context of a longer life expectancy. CKD reflects the occurrence of a premature aging process, similar to other chronic diseases [1]. Muscle mass is an important factor in determining the prognosis of these patients.

Myostatin, also called growth/differentiation factor-8, belongs to a transforming growth factor- $\beta$ superfamily and regulates the synthesis and degradation of skeletal muscle protein. Myostatin, which is mainly produced 
in muscle, suppresses growth in skeletal muscle and its inhibition leads to muscle hypertrophy. Myostatin levels are increased in patients with chronic skeletal muscle wasting diseases, such as CKD, chronic liver disease, or chronic heart failure [2-4]. Conversely, higher myostatin levels are associated with higher muscle mass in peritoneal dialysis (PD) patients [5]. It is not clear that myostatin levels are increased to prevent decreases in muscle mass in dialysis patients. Increased myostatin is associated with vascular inflammation and atherosclerotic changes $[6,7]$. However, there are few clinical data related to associated vascular calcification (VC) and no data regarding mortality available to date.

VC, a phenotype of vascular aging, is common in dialysis patients with longer dialysis histories and increases the risk of morbidity and mortality in these patients. Previous studies have shown that the abdominal aortic calcification (AAC) score on plain radiography has a very good correlation with the identification of coronary artery calcification and low bone mass, which are risk factors for mortality $[8,9]$. A previous human study reported that serum myostatin levels were negatively associated with AAC in older males [10]. However, no research has been conducted on the relationship between myostatin levels and AAC in dialysis patients at this time, to our knowledge. The aim of this study was therefore to assess the association between serum myostatin level and AAC in dialysis patients. In addition, we analyzed the relationship between serum myostatin level, muscle mass, and bone mineral density (BMD).

\section{Methods}

\section{Study design and patients}

We conducted a cross-sectional study at the Dong-A University Dialysis Center from March 2013 to September 2014. Patients who had been undergoing dialysis for at least six months and who were older than 20 years of age were enrolled. Those who had malignancies or active inflammatory diseases within the past three months were excluded. Enrolled patients were assessed based on plain radiographs. Hemodialysis (HD) patients were dialyzed three times per week through bicarbonate-based dialysate and polysulfone dialyzers (Fresenius, Bad Homburg, Germany). PD patients received four exchanges per day using a standard regimen (8 L/day).

This study was approved by the Dong-A University Institutional Review Board (approval no. DAUHIRB-17-011). Informed consent was obtained from all of the study participants. All data were anonymized and deidentified prior to analysis. Investigations were performed in accordance with the Declaration of Helsinki.

\section{Laboratory measurements}

Routine laboratory tests were performed using fasting blood samples in PD patients and collected blood prior to HD. Serum levels of hemoglobin, glucose, blood urea nitrogen, creatinine, albumin, calcium, phosphorus, alkaline phosphatase, C-reactive protein, parathyroid hormone (PTH), total cholesterol, and triglycerides were evaluated. Blood myostatin levels were analyzed using a commercially available competitive enzyme-linked immunosorbent assay (ELISA) kit (R\&D Systems Inc., Minneapolis, NM, USA) based on monoclonal antibodies raised against the recombinant factor. We classified patients into two groups according to the median value of myostatin, as follows: those with high myostatin levels $(\geq 5.0 \mathrm{ng} / \mathrm{mL})$ and those with low myostatin levels $(<5.0$ $\mathrm{ng} / \mathrm{mL}$ ). Fetuin-A, osteoprotegerin (OPG), and receptor activator of nuclear factor kappa B ligand (RANKL) levels were measured by ELISA (BioVendor Laboratory Medicine, Brno, Czech Republic). Fibroblast growth factor 23 (FGF-23) levels were measured using a Millipore FGF23 ELISA kit (EMD Millipore, Burlington, MA, USA). We measured testosterone and estrogen levels using a radioimmunoassay approach.

\section{Vascular calcification score on plain radiography}

Plain radiographs of the lateral lumbar spine were evaluated for AAC. AAC was scored using a previously reported method according to the location and degree of calcification in individual lumbar vertebral segments from L1 to L4 $[11,12]$. These scores were summarized in two ways. First, there was a composite score for an anteroposterior severity, which is calculated including aortic segment scores for both the anterior and posterior walls (maximum score: 24 points). Second, there was an affected segment score denoting the total number of aortic segments showing any level of calcification (maximum 
score: 4 points). It has been reported that an AAC score of five or more points out of 24 points increases the risk of cardiovascular disease [12-14]. Two nephrologists individually determined the AAC scores, and a consensus was achieved for all radiograph interpretations.

\section{Body composition assessment and BMD}

Twenty patients underwent an assessment of their body composition, either at the time of or within six months of blood sampling. Body composition was assessed using an X-scan Plus II body composition analyzer (Jawon Medical Co., Ltd., Gyeongsan, Korea). Appendicular skeletal muscle mass was defined as the sum of the lean masses for both arms and legs [15]. The appendicular skeletal muscle index was calculated as follows: appendicular skeletal muscle mass index (ASMI) = appendicular skeletal muscle mass/height ${ }^{2}$. It is expressed in kilograms per meter squared.

BMD was measured for the femur neck, total hip, and lumbar spine (L1-L4) by the means of dual-energy X-ray absorptiometry using a Discovery W scanner (Hologic Inc., Waltham, MA, USA). BMD results were obtained as a T-score; the T-score indicates the number of standard deviations (SDs) from the mean value of a healthy young adult population (age: $20-40$ years).

\section{Statistical analysis}

Data were expressed as means \pm SDs and medians (interquartile ranges) as appropriate. The subjects' characteristics were analyzed using a Student's $t$ test if

Table 1. Comparison of clinical characteristics in accordance with myostatin level

\begin{tabular}{|c|c|c|c|c|}
\hline Characteristic & Total $(n=71)$ & $\begin{array}{c}\text { Myostatin }<5.0 \mathrm{ng} / \mathrm{mL} \\
(\mathrm{n}=35)\end{array}$ & $\begin{array}{c}\text { Myostatin } \geq 5.0 \mathrm{ng} / \mathrm{mL} \\
(\mathrm{n}=36)\end{array}$ & $P$ value \\
\hline Age (yr) & $58.9 \pm 11.2$ & $62.6 \pm 9.1$ & $55.3 \pm 11.9$ & 0.005 \\
\hline Sex (male) & $39(54.9)$ & $14(40.0)$ & $25(69.4)$ & 0.013 \\
\hline Dialysis vintage (mo) & $33.0(18.0-62.0)$ & $39(18.0-67.0)$ & $28(18.0-61.0)$ & 0.633 \\
\hline Dialysis modality (PD) & $34(47.9)$ & $10(28.6)$ & $24(66.7)$ & 0.001 \\
\hline Diabetes mellitus & $41(57.7)$ & $21(60.0)$ & $20(55.6)$ & 0.705 \\
\hline Body mass index $\left(\mathrm{kg} / \mathrm{m}^{2}\right)$ & $22.9 \pm 3.3$ & $22.6 \pm 3.7$ & $23.1 \pm 2.8$ & 0.561 \\
\hline \multicolumn{5}{|c|}{ Calcification scores on plain radiograph } \\
\hline$A A C \geq 5$ points & $35(49.3)$ & $25(71.4)$ & $10(27.8)$ & $<0.001$ \\
\hline AAC scores & $4.0(0-9.0)$ & $6.0(3.0-11.0)$ & $1.5(0-5.8)$ & 0.001 \\
\hline Albumin (g/dL) & $3.9 \pm 0.4$ & $3.9 \pm 0.4$ & $3.9 \pm 0.3$ & 0.724 \\
\hline Calcium (mg/dL) & $8.9 \pm 0.7$ & $9.1 \pm 0.7$ & $8.8 \pm 0.7$ & 0.117 \\
\hline Phosphorus (mg/dL) & $5.4 \pm 1.7$ & $5.0 \pm 1.9$ & $5.7 \pm 1.5$ & 0.089 \\
\hline Alkaline phosphatase (IU/L) & 278.0 (199.0-374.0) & $270.0(198.0-327.0)$ & $280.5(200.5-406.8)$ & 0.683 \\
\hline C-reactive protein (mg/dL) & $0.2(0.1-0.5)$ & $0.3(0.1-0.7)$ & $0.1(0-0.4)$ & 0.005 \\
\hline Cholesterol (mg/dL) & $156.9 \pm 39.6$ & $158.3 \pm 37.7$ & $155.5 \pm 41.9$ & 0.763 \\
\hline Triglyceride (mg/dL) & $139.1 \pm 71.5$ & $147.5 \pm 79.2$ & $130.7 \pm 62.8$ & 0.330 \\
\hline Parathyroid hormone (pg/mL) & $354.0 \pm 231.6$ & $308.8 \pm 240.7$ & $397.9 \pm 216.8$ & 0.106 \\
\hline Fetuin-A ( $\mu \mathrm{g} / \mathrm{mL})$ & $244.9 \pm 73.2$ & $256.3 \pm 78.2$ & $233.7 \pm 67.2$ & 0.195 \\
\hline FGF-23 (pg/mL) & $1,653.7 \pm 1,551.0$ & $1,711.2 \pm 1,668.4$ & $1,598.0 \pm 1,452.0$ & 0.771 \\
\hline Osteoprotegerin (pmol/L) & $24.4 \pm 10.4$ & $26.1 \pm 10.6$ & $22.7 \pm 10.1$ & 0.171 \\
\hline RANKL (pmol/L) & $263.1(138.7-404.3)$ & $338.3(203.2-447.4)$ & $215.2(123.5-391.4)$ & 0.124 \\
\hline Myostatin (ng/mL) & $5.3 \pm 2.6$ & $3.3 \pm 1.1$ & $7.3 \pm 2.1$ & $<0.001$ \\
\hline Estrogen (pg/mL) & $20.3 \pm 16.9$ & $21.7 \pm 19.3$ & $17.5 \pm 10.8$ & 0.530 \\
\hline Testosterone (pg/mL) & $3.8 \pm 1.5$ & $3.7 \pm 1.7$ & $3.8 \pm 1.4$ & 0.881 \\
\hline ASM/height ${ }^{2}$ & $8.1 \pm 1.2$ & $7.9 \pm 1.0$ & $8.6 \pm 1.4$ & 0.210 \\
\hline
\end{tabular}

Data are presented as mean \pm standard deviation, number (\%), or median (interquartile range).

AAC, abdominal aortic calcification; ASM, appendicular skeletal muscle mass; FGF-23, fibroblast growth factor 23; PD, peritoneal dialysis; RANKL, receptor activator of nuclear factor kappa B ligand. 
normally distributed or with the Mann-Whitney $U$ test if the distribution was skewed. A chi-squared test was used to compare categorical data between the two groups. Correlation analysis was performed using Spearman's rank correlation. To independently evaluate the factors associated with blood myostatin levels, univariate and multivariate logistic regression analyses were performed. A $P$ value of $<0.05$ was considered to be statistically significant. All statistical calculations were performed using the SPSS version 18.0 (SPSS Inc., Chicago, IL, USA).

\section{Results}

\section{Clinical characteristics were in accordance with myostatin level}

In all, 71 dialysis patients were enrolled in this study. The baseline characteristics of these participants are shown in Table 1 . The mean age was $58.9 \pm 11.2$ years, and 39 patients $(54.9 \%)$ were male. Our study included 37 HD patients and 34 PD patients, and the median myostatin level in these individuals was $5.0 \mathrm{ng} / \mathrm{mL}$.

Patients with low myostatin levels were typically older and included a lower proportion of males and PD patients among them (Table 1). The proportion of patients with an AAC score of five or more points was significantly higher in the group with low myostatin levels than in the group with high myostatin levels. No significant differences in the laboratory findings including PTH, fetuin-A, FGF-23, RANKL, and OPG results, were found between the two groups.

Clinical characteristics were in accordance with $A A C$ score on plain radiography

The median myostatin level for patients with AAC

Table 2. Comparison of clinical characteristics in accordance with AAC score on plain radiography

\begin{tabular}{|c|c|c|c|}
\hline Characteristic & AAC $<5$ points $(n=36)$ & AAC $\geq 5$ points $(n=35)$ & $P$ value \\
\hline Age (yr) & $55.0 \pm 12.6$ & $62.9 \pm 7.7$ & 0.002 \\
\hline Sex (male) & $23(63.9)$ & $16(45.7)$ & 0.124 \\
\hline Dialysis vintage (mo) & $21.5(15.3-55.3)$ & $41.0(32.0-84.0)$ & 0.633 \\
\hline Dialysis modality (PD) & $22(61.1)$ & $12(34.3)$ & 0.024 \\
\hline Diabetes mellitus & $20(55.6)$ & $21(60.0)$ & 0.705 \\
\hline Body mass index $\left(\mathrm{kg} / \mathrm{m}^{2}\right)$ & $23.1 \pm 3.1$ & $22.6 \pm 3.5$ & 0.467 \\
\hline \multicolumn{4}{|c|}{ Calcification scores on plain radiograph } \\
\hline AAC scores & $0(0-2.8)$ & $9.0(6.0-15.0)$ & 0.001 \\
\hline Albumin (g/dL) & $3.9 \pm 0.4$ & $3.9 \pm 0.4$ & 0.876 \\
\hline Calcium (mg/dL) & $8.8 \pm 0.8$ & $9.0 \pm 0.7$ & 0.265 \\
\hline Phosphorus (mg/dL) & $5.3 \pm 1.4$ & $5.5 \pm 2.0$ & 0.709 \\
\hline Alkaline phosphatase (IU/L) & $280.5(210.0-372.3)$ & $274.0(168.0-397.0)$ & 0.683 \\
\hline C-reactive protein (mg/dL) & $0.1(0.0-0.4)$ & $0.3(0.1-0.5)$ & 0.005 \\
\hline Cholesterol (mg/dL) & $160.0 \pm 43.7$ & $153.7 \pm 35.3$ & 0.509 \\
\hline Triglyceride (mg/dL) & $130.7 \pm 63.7$ & $147.4 \pm 78.5$ & 0.333 \\
\hline Parathyroid hormone (pg/mL) & $351.9 \pm 206.8$ & $356.1 \pm 247.7$ & 0.940 \\
\hline Fetuin-A ( $\mu \mathrm{g} / \mathrm{mL})$ & $248.5 \pm 78.1$ & $241.2 \pm 68.7$ & 0.676 \\
\hline FGF-23 (pg/mL) & $1,275.2 \pm 1,269.1$ & $2,068.9 \pm 1,737.8$ & 0.042 \\
\hline Osteoprotegerin (pmol/L) & $21.8 \pm 9.5$ & $27.0 \pm 10.8$ & 0.036 \\
\hline RANKL (pmol/L) & 306.2 (159.6-474.6) & $215.6(125.0-354.6)$ & 0.124 \\
\hline Myostatin (ng/mL) & $6.4 \pm 2.9(5.8)$ & $4.2 \pm 1.7(4.1)$ & $<0.001$ \\
\hline Estrogen (pg/mL) & $22.9 \pm 23.4$ & $18.5 \pm 11.0$ & 0.554 \\
\hline Testosterone (pg/mL) & $4.1 \pm 1.4$ & $3.2 \pm 1.2$ & 0.082 \\
\hline ASM/height ${ }^{2}$ & $8.3 \pm 1.4$ & $8.0 \pm 1.1$ & 0.502 \\
\hline
\end{tabular}

Data are presented as mean \pm standard deviation, number (\%), median (interquartile range), or mean \pm standard deviation (median).

AAC, abdominal aortic calcification; ASM, appendicular skeletal muscle mass; FGF-23, fibroblast growth factor 23; PD, peritoneal dialysis; RANKL, receptor activator of nuclear factor kappa B ligand. 
scores of five or more points was $4.1 \mathrm{ng} / \mathrm{mL}$, whereas that for patients with AAC scores of less than five points was $5.8 \mathrm{ng} / \mathrm{mL}$ (Table 2). Patients with AAC scores of five or more points were typically older with a higher proportion of HD patients with longer dialysis timelines as compared with those with AAC scores of less than five points. Among several bone markers, the levels of FGF-23 and OPG were significantly higher among patients with AAC scores of five or more points than those with AAC scores of less than five points. However, no significant differences in laboratory findings including PTH, fetuin-A, and RANKL were found between the groups.

\section{Comparison between myostatin levels and T-scores for $B M D$}

We compared the mean T-score for BMD according to

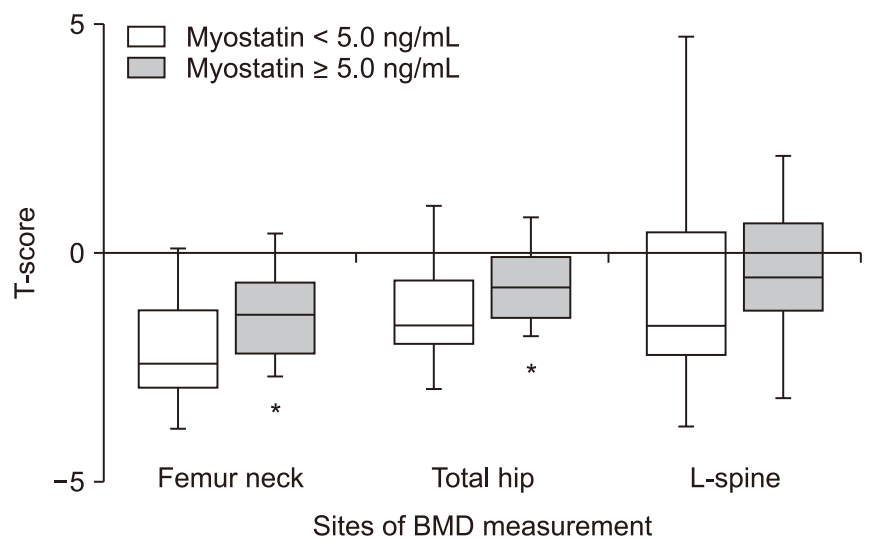

Figure 1. Comparison of T-scores for different sites of bone mineral density (BMD) measurement in accordance with myostatin level $(n=71)$. Statistical differences between two groups were determined using the Student's $t$ test. $* P<0.05$.

Table 3. Correlations of plasma myostatin level and AAC score with various parameters

\begin{tabular}{|c|c|c|c|c|}
\hline \multirow{2}{*}{ Variable } & \multicolumn{2}{|c|}{ Myostatin level (ng/mL) } & \multicolumn{2}{|c|}{ AAC score } \\
\hline & $\mathrm{r}$ & $P$ value & $\mathrm{r}$ & $P$ value \\
\hline Age (yr) & -0.385 & 0.001 & 0.390 & 0.001 \\
\hline Dialysis vintage $(\mathrm{mo})$ & -0.123 & 0.308 & 0.386 & 0.001 \\
\hline Body mass index $\left(\mathrm{kg} / \mathrm{m}^{2}\right)$ & 0.123 & 0.208 & -0.106 & 0.381 \\
\hline \multicolumn{5}{|c|}{ Calcification score on plain radiograph } \\
\hline AAC score & -0.426 & $<0.001$ & & \\
\hline \multicolumn{5}{|l|}{ T-score on bone mineral density } \\
\hline Total hip & 0.409 & 0.002 & -0.254 & 0.057 \\
\hline Femur neck & 0.423 & 0.005 & -0.306 & 0.046 \\
\hline Lumbar spine & 0.249 & 0.036 & -0.114 & 0.345 \\
\hline Albumin (g/dL) & -0.142 & 0.237 & 0.063 & 0.603 \\
\hline Calcium (mg/dL) & -0.199 & 0.096 & 0.118 & 0.326 \\
\hline Phosphorus (mg/dL) & 0.255 & 0.032 & 0.048 & 0.692 \\
\hline Alkaline phosphatase (IU/L) & 0.048 & 0.692 & 0.066 & 0.587 \\
\hline C-reactive protein (mg/dL) & -0.318 & 0.007 & 0.252 & 0.034 \\
\hline Cholesterol (mg/dL) & -0.089 & 0.460 & -0.168 & 0.161 \\
\hline Triglyceride $(\mathrm{mg} / \mathrm{dL})$ & -0.173 & 0.151 & 0.066 & 0.589 \\
\hline Parathyroid hormone $(\mathrm{pg} / \mathrm{mL})$ & 0.289 & 0.015 & 0.035 & 0.774 \\
\hline Fetuin-A $(\mu g / m L)$ & -0.076 & 0.531 & -0.016 & 0.894 \\
\hline FGF-23 (pg/mL) & -0.028 & 0.825 & 0.282 & 0.023 \\
\hline Osteoprotegerin (pmol/L) & -0.172 & 0.152 & 0.235 & 0.048 \\
\hline RANKL (pmol/L) & -0.214 & 0.196 & -0.091 & 0.588 \\
\hline Estrogen $(\mathrm{pg} / \mathrm{mL})$ & 0.019 & 0.919 & -0.049 & 0.795 \\
\hline Testosterone (pg/mL) & 0.207 & 0.265 & -0.217 & 0.240 \\
\hline Myostatin $(\mathrm{ng} / \mathrm{mL})$ & & & -0.426 & $<0.001$ \\
\hline ASM/height ${ }^{2}$ & 0.516 & 0.020 & -0.074 & 0.758 \\
\hline
\end{tabular}

AAC, abdominal aortic calcification; ASM, appendicular skeletal muscle mass; FGF-23, fibroblast growth factor 23; RANKL, receptor activator of nuclear factor kappa $B$ ligand. 
myostatin level. The mean T-score for BMD measured at the femur neck was relatively lower than that for BMD assessed at the total hip or lumbar spine $(-1.7 \pm 1.0,-1.0 \pm$ 1.0 , and $-0.6 \pm 1.7$, respectively). The T-scores for BMD measurements taken at the femur neck and total hip in patients with low myostatin levels were significantly lower than those in patients with high myostatin levels; however, there were no significant differences in the Tscore for BMD measurements taken at the lumbar spine between the two groups (Fig. 1).

\section{Association between myostatin level, AAC score, and clinical parameters}

A significant correlation was observed between myostatin level, AAC score, and related parameters (Table $3)$. AAC score showed a significantly negative correlation with myostatin level $(\mathrm{r}=-0.426, P<0.001$; Fig. 2). Meanwhile, a positive relationship between ASMI value and myostatin level was found $(r=0.516, P=0.020$; Fig. 3). T-scores for BMD measured at the total hip, femur neck, and lumbar spine all had a significantly positive association with myostatin level. No significant correlations were observed between myostatin level and OPG, FGF23, fetuin-A, and RANKL, respectively.

\section{Independent factors associated with myostatin level and AAC score}

On univariate logistic regression analysis, age, sex, dialysis modality, and AAC score of five points or more were found to be associated with high myostatin levels. To confirm the predictors for myostatin level, we performed a multiple logistic regression analysis (Table 4). Lower myostatin levels were independently associated with higher AAC scores following adjustment for age, sex, diabetes mellitus, dialysis vintage, dialysis modality, and OPG level. In addition, higher AAC scores were indepen-

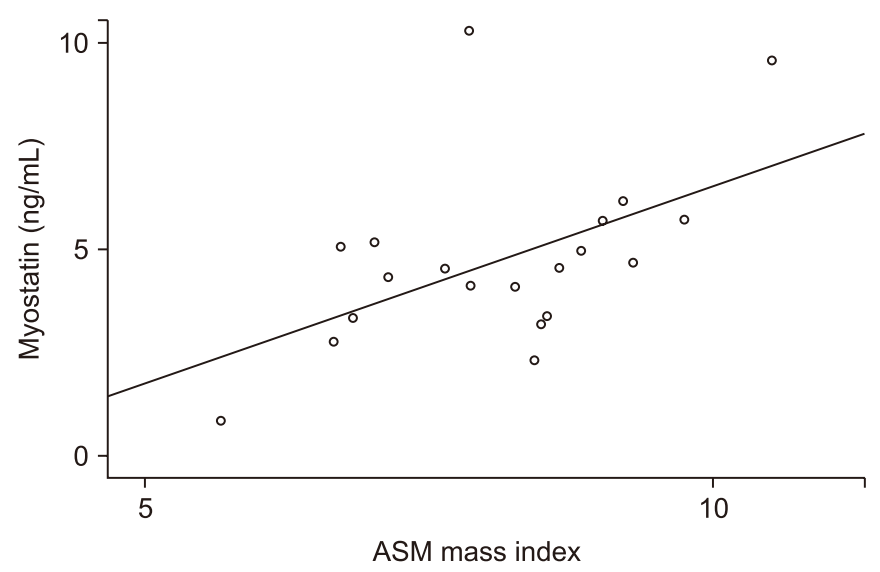

Figure 3. Correlation between height-adjusted skeletal muscle mass and serum myostatin level $(r=0.516, P=0.020 ; n=20)$. ASM, appendicular skeletal muscle.

Table 4. Independent factors associated with high myostatin levels (myostatin $\geq 5.0 \mathrm{ng} / \mathrm{mL}$ )

\begin{tabular}{lcccc}
\hline \multicolumn{1}{c}{ Variable } & Univariate & $P$ value & Multivariate & $P$ value \\
\hline Age $(\mathrm{yr})$ & $0.33(0.13-0.88)$ & 0.026 & $0.28(0.06-1.38)$ & 0.118 \\
Sex (male) & $3.41(1.28-9.08)$ & 0.014 & $5.92(1.44-24.33)$ & 0.014 \\
Diabetes mellitus & $0.83(0.33-2.14)$ & 0.705 & $0.55(0.14-2.07)$ & 0.375 \\
Dialysis vintage (mo) & $0.67(0.26-1.72)$ & 0.408 & $3.18(0.59-17.10)$ & 0.178 \\
Dialysis modality (PD) & $5.00(1.82-13.71)$ & 0.002 & $8.14(1.95-33.91)$ & 0.004 \\
Osteoprotegerin (pmol/L) & $0.94(0.37-2.40)$ & 0.904 & $1.60(0.37-6.88)$ & 0.526 \\
AAC score $\geq 5$ points & $0.15(0.06-0.43)$ & $<0.001$ & $0.16(0.03-0.75)$ & 0.020 \\
\hline
\end{tabular}

Data are presented as odds ratio (95\% confidence interval).

AAC, abdominal aortic calcification; PD, peritoneal dialysis. 
dently associated with lower myostatin levels (Supplementary Table 1; available online).

\section{Discussion}

VC is a well-known complication of end-stage renal disease. Several markers such as OPG, RANKL, fetuin$\mathrm{A}$, and FGF-23 act as VC inhibitors or inducers, but the role of myostatin as a VC inhibitor or inducer is unclear. In this study, myostatin levels were negatively associated with AAC scores on lateral lumbar spine radiographs. Patients with high myostatin levels had lower AAC scores than did those with low myostatin levels, indicating potentially favorable effects on VC in dialysis patients. Szulc et al [10] reported previously in the Structure of the Aging Men's Bones (STRAMBO) study that older males with AAC have lower myostatin levels than those without AAC. Generally, the expression of myostatin and the severity of vascular damage show a positive correlation. In mice with myostatin deficiencies, bone marrow-derived mesenchymal stem cells showed increased osteogenic differentiation through the regulation of osteogenic factors [16]. Lesser muscle mass and greater fat mass are associated with a higher prevalence of VC [17]. In this regard, VC may frequently occur when muscle mass decreases. It is necessary to monitor the continuous changes in myostatin levels relating to skeletal muscle mass effects on VC incidence or progression in dialysis patients.

Muscle wasting with high myostatin levels is found in the general population $[18,19]$. Blood myostatin levels are increased according to the decline of renal function [20] and myostatin may be a main mediator of CKD-related sarcopenia. However, we found that higher serum myostatin levels are associated with higher muscle mass in dialysis patients. Similar data findings regarding muscle mass and myostatin level have previously been reported in PD patients [5]. In our study, skeletal muscle mass calculated using the ASMI approach was positively associated with myostatin level, indicating that myostatin level is partially determined by muscle mass in dialysis patients. Myostatin is expressed in the heart and adipose tissue but is mainly synthesized and excreted by skeletal muscles [21-23]. It is presumed that myostatin production itself can be decreased when muscle mass is decreased in dialysis patients. It is noteworthy that myostatin levels are high enough in dialysis patients, as these individuals are advanced CKD patients. Therefore, lower myostatin levels may reflect relatively decreased intact muscle mass, which synthesizes and excretes myostatin in dialysis patients. This finding may also represent another reverse epidemiology which higher myostatin levels indicate higher muscle mass, such as in the case of higher cholesterol levels indicating good nutritional status in dialysis patients [24]. In comparison, higher levels of both markers are harmful in predialysis CKD patients. However, decreasing myostatin levels without changing muscle mass by with exercise or high flux dialysis was found to be beneficial for improving muscle power in HD patients $[25,26]$. The exact roles of myostatin and its mechanisms related to muscle mass are unknown. Further prospective studies are needed to validate these findings in predialysis and dialysis CKD patients. It is necessary to monitor myostatin levels from predialysis periods through longterm dialysis periods.

In this study, the BMD at the femur neck was relatively lower than that at the total hip or lumbar spine. Since BMD at the spine is disturbed by artifacts like VC and peritoneal fluid in dialysis patients, overall bone density can be overestimated [27]. For this reason, errors may occur in interpreting the BMD in dialysis patients. Nonetheless, BMD is still a useful method for diagnosing bone loss and predicting fracture, even in dialysis patients. VC is related with osteoblast-like cell differentiation from vascular smooth muscle cells and calcium may precipitate toward vessels instead of bone, especially in dialysis patients with adynamic bone disease. Therefore, $\mathrm{VC}$ is closely associated with BMD. In addition, the relationship between bone and muscle is still unclear. In a previous study with myostatin-knockout mice, muscle mass and BMD at the femur reportedly increased as compared with in control mice [28]. In a study from China, total myostatin levels were positively associated with BMD, although they no longer were correlated with BMD after adjusting for lean body mass [29]. In our study, patients with high myostatin levels had higher T-scores when BMD was measured at the femur neck or total hip. Like the connection of myostatin level with muscle mass in dialysis patients, the relationship between T-score for BMD and muscle mass is assumed. We suspect that myostatin does not decrease AAC or increase bone density. We suggest that higher myostatin levels simply reflect muscle mass and relatively maintained muscle mass may be re- 
lated to lower AAC values and higher T-scores for BMD in dialysis patients. Further studies are warranted to identify any cross-linkages between bone, muscle, and VC.

According to this study, PD may be a better modality than HD for maintaining high myostatin levels. However, PD patients enrolled in this study had significantly lower dialysis durations ( $34.5 \pm 27.7$ vs. $55.7 \pm 40.8$ months; $P=$ 0.012), reflecting smaller periods of time until myostatin levels decreased compared to HD patients. Therefore, further prospective studies are necessary to confirm the effect of dialysis modality on myostatin level.

This study had some limitations. First, the power of this study was limited because of the relatively small number of participants. Second, the design of the study was cross-sectional. Lastly, muscle mass was measured in only a few patients and muscle strength or physical performance were not measured.

To our knowledge, this is the first report to demonstrate that myostatin level is associated with AAC score, muscle mass, and BMD in patients undergoing dialysis. Further prospective studies are needed to validate these findings including in large cohorts.

\section{Conflicts of interest}

All authors have no conflicts of interest to declare.

\section{Acknowledgments}

This study was supported by the National Research Foundation of Korea (2017R1C1B5016636).

\section{Authors' contributions}

Won Suk An conceived and designed the study. Su Mi Lee, Seong Eun Kim, Ji Young Lee, Hyo Jin Jeong, Young Ki Son, and Won Suk An performed the study. Su Mi Lee, Seong Eun Kim, and Won Suk An participated in the preparation of the manuscript, statistical analysis, and data interpretation. All authors have read and approved the final manuscript.

\section{References}

[1] Kooman JP, Dekker MJ, Usvyat LA, et al. Inflammation and premature aging in advanced chronic kidney disease. Am J
Physiol Renal Physiol 2017;313:F938-F950.

[2] Verzola D, Procopio V, Sofia A, et al. Apoptosis and myostatin mRNA are upregulated in the skeletal muscle of patients with chronic kidney disease. Kidney Int 2011;79:773782.

[3] García PS, Cabbabe A, Kambadur R, Nicholas G, Csete M. Brief-reports: elevated myostatin levels in patients with liver disease: a potential contributor to skeletal muscle wasting. Anesth Analg 2010;111:707-709.

[4] Lenk K, Schur R, Linke A, et al. Impact of exercise training on myostatin expression in the myocardium and skeletal muscle in a chronic heart failure model. Eur J Heart Fail 2009;11:342-348.

[5] Yamada S, Tsuruya K, Yoshida H, et al. Factors associated with the serum myostatin level in patients undergoing peritoneal dialysis: potential effects of skeletal muscle mass and vitamin D receptor activator use. Calcif Tissue Int 2016;99:13-22.

[6] Verzola D, Milanesi S, Bertolotto M, et al. Myostatin mediates abdominal aortic atherosclerosis progression by inducing vascular smooth muscle cell dysfunction and monocyte recruitment. Sci Rep 2017;7:46362.

[7] Verzola D, Barisione C, Picciotto D, Garibotto G, Koppe L. Emerging role of myostatin and its inhibition in the setting of chronic kidney disease. Kidney Int 2019;95:506-517.

[8] Lee SM, Lee HW, Son YK, Kim SE, An WS. Abdominal aortic calcification score among several vascular calcification scores of plain radiograph is the most reliable predictor of severe coronary artery calcification in dialysis patients. Ren Fail 2017;39:729-735.

[9] Nam HS, Lee SM, Jeong EG, et al. Vascular calcification on plain radiographs is related with the severity of lesions detected by coronary angiography in dialysis patients. Tohoku J Exp Med 2015;235:135-144.

[10] Szulc P, Hofbauer LC, Rauner M, Goettsch C, Chapurlat R, Schoppet M. Serum myostatin levels are negatively associated with abdominal aortic calcification in older men: the STRAMBO study. Eur J Endocrinol 2012;167:873-880.

[11] Adragao T, Pires A, Lucas C, et al. A simple vascular calcification score predicts cardiovascular risk in haemodialysis patients. Nephrol Dial Transplant 2004;19:1480-1488.

[12] Honkanen E, Kauppila L, Wikström B, et al; CORD study group. Abdominal aortic calcification in dialysis patients: results of the CORD study. Nephrol Dial Transplant 2008; 23:4009-4015.

[13] Verbeke F, Van Biesen W, Honkanen E, et al. Prognostic 
value of aortic stiffness and calcification for cardiovascular events and mortality in dialysis patients: outcome of the calcification outcome in renal disease (CORD) study. Clin J Am Soc Nephrol 2011;6:153-159.

[14] An WS, Son YK. Vascular calcification on plain radiographs is associated with carotid intima media thickness, malnutrition and cardiovascular events in dialysis patients: a prospective observational study. BMC Nephrol 2013;14:27.

[15] Heymsfield SB, Smith R, Aulet M, et al. Appendicular skeletal muscle mass: measurement by dual-photon absorptiometry. Am J Clin Nutr 1990;52:214-218.

[16] Hamrick MW, Shi X, Zhang W, et al. Loss of myostatin (GDF8) function increases osteogenic differentiation of bone marrow-derived mesenchymal stem cells but the osteogenic effect is ablated with unloading. Bone 2007;40: 1544-1553.

[17] Jensky NE, Criqui MH, Wright CM, Wassel CL, Alcaraz JE, Allison MA. The association between abdominal body composition and vascular calcification. Obesity (Silver Spring) 2011;19:2418-2424.

[18] Schuelke M, Wagner KR, Stolz LE, et al. Myostatin mutation associated with gross muscle hypertrophy in a child. $N$ Engl J Med 2004;350:2682-2688.

[19] Seibert MJ, Xue QL, Fried LP, Walston JD. Polymorphic variation in the human myostatin (GDF-8) gene and association with strength measures in the Women's Health and Aging Study II cohort. J Am Geriatr Soc 2001;49:1093-1096.

[20] Yano S, Nagai A, Isomura M, et al. Relationship between blood myostatin levels and kidney function: Shimane CoHRE Study. PLoS One 2015;10:e0141035.

[21] McPherron AC, Lawler AM, Lee SJ. Regulation of skeletal muscle mass in mice by a new TGF-beta superfamily mem- ber. Nature 1997;387:83-90.

[22] Shan T, Liang X, Bi P, Kuang S. Myostatin knockout drives browning of white adipose tissue through activating the AMPK-PGCl $\alpha$-Fndc5 pathway in muscle. FASEB J 2013;27: 1981-1989.

[23] Sharma M, Kambadur R, Matthews KG, et al. Myostatin, a transforming growth factor-beta superfamily member, is expressed in heart muscle and is upregulated in cardiomyocytes after infarct. J Cell Physiol 1999;180:1-9.

[24] Kalantar-Zadeh K, Block G, Humphreys MH, Kopple JD. Reverse epidemiology of cardiovascular risk factors in maintenance dialysis patients. Kidney Int 2003;63:793-808.

[25] Kopple JD, Cohen AH, Wang H, et al. Effect of exercise on mRNA levels for growth factors in skeletal muscle of hemodialysis patients. J Ren Nutr 2006;16:312-324.

[26] Han DS, Chen YM, Lin SY, et al. Serum myostatin levels and grip strength in normal subjects and patients on maintenance haemodialysis. Clin Endocrinol (Oxf) 2011;75:857863.

[27] Pocock N. Use of dual energy X-ray absorptiometry, the trabecular bone score and quantitative computed tomography in the evaluation of chronic kidney disease-mineral and bone disorders. Nephrology (Carlton) 2017;22 Suppl 2: 19-21.

[28] Hamrick MW. Increased bone mineral density in the femora of GDF8 knockout mice. Anat Rec A Discov Mol Cell Evol Biol 2003;272:388-391.

[29] Wu LF, Zhu DC, Wang BH, et al. Relative abundance of mature myostatin rather than total myostatin is negatively associated with bone mineral density in Chinese. J Cell Mol Med 2018;22:1329-1336. 